\title{
Introduction to the Proceedings of "Complexity-disorder" days
}

\author{
Jean-Claude Serge Levy ${ }^{1 *}$ \\ ${ }^{1}$ MPQ, UMR CNRS 7162, Physics Department, University Paris Diderot, 75013 Paris, France
}

\begin{abstract}
The common use of several disciplines for the realization of practical purposes and their usual coworking favour such meetings involving different disciplines. Some old and recent successful examples of effective interferences between different disciplines are reported in order to evidence the potential fruitfulness of this process. The theme complexity and disorder are shown to be a central point of convergence between disciplines shown from the observation of past and future projects.
\end{abstract}

\section{The necessity of pluri-disciplinary meetings on complexity and disorder.}

While the present organization of sciences requires a continuously growing complexity of labelling indices, i.e. a higher and higher level of specialization which corresponds indeed to a higher and higher level of social structuration, as already both observed and predicted a long time ago [1], many effective constraints lead to introduce also more general meetings, pluri-disciplinary ones as these "complexity-disorder" meetings here reported. This unexpected balance effect has several reasons, several effective constraints which are analysed below.

\subsection{The need for pluri-disciplinary meetings}

A first reason for introducing mixed meetings comes from the observation of the common use of complementary techniques in the realization of practical projects. When developing any enterprise or idea, different experts, different technical experiences are required. And the advices of these experts cannot be used, cannot be integrated just by means of a simple serial approach or by means of independent parallel developments. Experts must talk together, work together, interfere, in order to avoid possible misunderstandings and conflicts which occur within enterprises in the lack of direct contacts between experts [2]. Finally, in order to reach a realistic goal, these experts must build together a common project which must be consistent from all viewpoints in spite of its complexity. This is the reason for so numerous meetings and video conferences between different disciplines. This obvious and general evidence of the need for pluri-disciplinary meetings has several strong consequences. It proves the necessity and existence of such practical goal-oriented meetings. Moreover, this frequent and fruitful use means that such frequent meetings do not need a practical goal to occur.
This very simple picture of a basic practical, concrete action introduces practically some powerful concepts of physics, such as "interference", and of electronics and informatics such as "feedback" which are also shown here to be meaningful and efficient far from their basic field, linear and non-linear physics. It also introduces the unavoidable complexity of reality and of social division which must be overpassed in some way according to circumstances in order to achieve a practical goal.

These first remarks already point out the effective high level of both natural and required imbrication between disciplines which is necessary for achieving a realistic project. The same need of interference between different disciplines is also clear in politics. An approximate quotation of Mao Zedong means that the complexity of reality requires a multiplicity of viewpoints to be understood. This long-distance view is obviously a basic stone for introducing pluri-disciplinary meetings and specially about complexity.

Similar imbrications of different specialities also occur between partners of sport teams for instance. Within a team each sportsman or sportswoman must be aware of what his or her partners can do, and are used to do at any time, in order to develop a fruitful collective action. One may add that these sport partners also need to know the competences of their opponents in order to play efficiently and to reach the goals of their own team!

Rather similarly a modern philosopher could not speak of feelings like happiness [3] without taking into account not only his own experience and the experience of other philosophers during the whole history and all around the world, but also the observations of modern neurosciences on perception and feelings! It is necessary to account for other observations in order to obtain now a fully consistent realistic representation which can convince people. Even most famous and ancient disciplines cannot forget different disciplines and drive alone anymore! Reversely, the famous Hegel's Aesthetics [4] puts opera at a top level among all arts since it appeals most of human senses. This feeling is now validated by neural

* Corresponding author: jean-claude.levy@univ-paris-diderot.fr 
observations which evidence such automatic synthesis between sensations within the brain.

The interesting principle of falsification introduced by Popper [5] for classifying scientific results and theories leads not only to check the validity of an observation or a theory within its own field, but also to check its validity domain, i.e. the domains where it can be applied and where it cannot be applied, even out of its own field. In other words, this principle requires a validation process or an invalidation process from other specialists in order to allow the building of a consistent tree of knowledge. Quite obviously such a validation process means the effective occurrence of pluri-disciplinary meetings, and finally of a very collective inter-disciplinary work in order to check this full consistency. This very constitution of knowledge requires a global interest and practically pluri-disciplinary meetings.

Another simple reason for introducing mixed meetings is the very basic need of communication of specialists with other people without being only restricted to communication with a highly focused cast of high-level specialists. There is a relative basic question about this possibility: can specialists go through their own borders? Can specialists interest students or even the layman to their specialities? The previous arguments show that for social exchanges between different disciplines, such linguistic requirements are practically required, since translators would be aware of both disciplines. So, it is useful to take advantage of this practical pressure and to introduce early and often such social meetings between scientists and active people in order for all of them to be trained practically to such exchanges.

In a highly specialized meeting, the communication between specialists is necessarily elusive, since specialists must both develop the interest of their colleagues for their own work and save their original place within this scientific community against virtual concurrent colleagues. This original communication status induces strong limits to such a formal linguistic exchange with both a few attractive clear points and large undescribed zones. A pluri-disciplinary meeting overcomes this difficulty by the requirement to be understood in simple plain words by most of the whole audience. And, reversely this open language is also useful even for specialists themselves because of its clarity.

A complementary argument lies in the fact that specialists, as well as other human beings must always satisfy a very large number of different duties in their everyday life, and are obviously used in their own common life to face the full complexity of reality! So, their minds must be already well trained to such free exchanges which happen in the basic real life. From this point of view, pluri-disciplinary meetings give to the participants, these high-level specialists, the opportunity to free their own usual internal energy in simple ways.

A similar argument consists in looking at the whole life of a scientist. During his or her scientific life, about forty years, a scientist has a lot of times the opportunity of being interested in other fields than its own speciality, and quite numerous scientists follow such indirect ways, at least during some time. This multiplicity of interests, this complexity of the human mind is reached at the individual level! So, a scientist has naturally many interests in different topics which can be easily developed in common meetings.

To end the loop, it must be added that finally the goal of specialists consists in discovering new applications, new concepts which can be used widely, not only by pure specialists but by common people. In this way such large meetings open the path for this interesting communication with a quite larger community, in order to feel the needs and the interests of the whole human community, what remains a necessary task for specialists. So pluridisciplinary meetings are quite necessary in order to refresh from time to time the scientific community which otherwise would become closed in its own highly focused community.

The complexity of reality was often underlined here. So, it is useful now to look more carefully at complexity and disorder from different discipline viewpoints, and specially to disorder where complexity reaches its highest level.

\subsection{The relevance of complexity and disorder as one unifying theme}

The very theme complexity and disorder sounds quite justified for introducing such pluri-disciplinary works since this theme is not only common at all disciplines as already observed previously, but also this theme lies just at the common border between "hard" sciences and "human sciences.

Hard sciences try to deal with simple basic natural phenomena but cannot neglect the more complex ones which effectively occur. So, complexity lies within the field of hard sciences. Noise is quite observable and thus also belongs to the field of hard sciences. In a quite similar way, hard sciences which are devoted to complex human problems cannot neglect phenomena with more or less randomness as they also appear in the real world. This randomness belongs also to the field of hard sciences and is the basis for quite numerous statistical theories in different disciplines.

The practical, concrete and recent introduction of complexity and disorder within scientific themes was strongly linked with the occurrence of modern rapid quenching techniques which allow to fix definitely the instantaneous disorder of a liquid and then to observe its glassy properties in a quite general way for all materials [6]. Such observations had many fruitful consequences in physics, with the production of new materials such as metallic glasses and quasicrystals, the observation of new properties such as spin glasses. Finally, this extended work on disorder leads to a quite rich unexpected picture as recently shown [7]. This deep richness can lead to many practical applications and so becomes so attractive.

For human sciences, complexity and disorder are basic unavoidable features which were well known from their very beginning, and require a special care which is achieved by means of sophisticated scientific tools. This common border of complexity and disorder is a strong 
reason for long time collaboration between hard sciences and human sciences, with both common tools and common ideas, approaches. Reversely, it sounds that it could be useful now to read in the other way, what could human sciences with their long experience of complexity bring to hard sciences?

Moreover, this remark of a common border between hard sciences and human sciences also means that at the beginning of human culture, when the level of knowledge was very low for all sciences or disciplines, hard sciences and human sciences were very close, so close that strong connections were very active. Later, hard sciences knew a large success in explaining more and more simple things, and specialization appeared. The splitting of sciences into several disciplines was a necessary consequence of the abundance of fruitful topics. Human and social sciences appeared later as developed sciences, at the end of the nineteenth century. And now naturally the common interest of hard sciences and human sciences for more complex situations arises. So, now some mixed meetings on complexity and disorder are useful at least to detect what can be done together between so different disciplines.

The best proof of efficiency of such practices comes of course from real experiments and observations. Such historical experiments involving different disciplines in order to obtain a better understanding of nature started very early in the history of ideas and had quite numerous applications in many fields. This history goes on now. So, it sounds here quite useful to give a very brief review of these long scientific exchanges through the whole human history, even if this summary is here quite restrictive by principle and quite far from completion. It is just done here to show that at least a few main such interdisciplinary experiments already occurred. Such a summary evidences this more or less forgotten view of a common interest between so different sciences.

\section{A few historical examples of interdisciplinary contributions}

\subsection{Some old examples}

During the famous Greek golden age, numerous exceptional philosophers were also reported to be simultaneously excellent mathematicians and physicists $[8,9]$, as expected in these early times of common disciplines. And their results are still active and interesting nowadays!

Quite later, the modern era developed progressively a fine structure of observation and experience after the appealing evidence of numerous new discoveries of new practical tools such as relatively modern and stable ships like "Caravels" which enabled travellers to face tempests on the sea and so, to reach new worlds, new human civilizations, giving the path to more and more attractive discoveries. In this general exciting framework, linking ideas and experiments, Francis Bacon, a philosopher, early discussed about gravity and even proposed to measure the weight of an object at different places in order to prove the long-distance effect of attraction and gravity
[10] and so to understand the origin of gravity. This early "philosophical" work introduced a new unfinished decisive step in the framework of complexity, i.e. longdistance interactions, still an active field.

Later an apple fell upon Newton. This simple triggering fact motivated his whole research on the law of universal attraction [11], a link between physics and astronomy, a further step in the way introduced by Bacon. This new strong discovery was of a similar general nature as the old ones obtained in the early Hellenistic times [8,9], i.e. mixing philosophy, observation and mathematical treatment in order to obtain a global view of natural events. This law of dynamics mixed space and time and so was also the beginning of modern physics and mathematics, by introducing new problems of derivation and integration.

Another interesting point of useful convergence between disciplines, here biology and physics, is the case of animal electricity as observed in electric eels which were early known as effective "tasers". The analysis of the constitution of such electric eels which were given to the scientific community by Alexander von Humboldt in 1800 seems to have led Volta to the discovery of efficient batteries. This biomimetic application well illustrates this state of mixed sciences. It must be added that biomimetics remains an active field among several other crossdisciplinary domains [12].

\subsection{Some more recent mixed concepts}

\subsubsection{Mixed concepts in physics, electronics and informatics}

Another large jump through the history of sciences leads us now to consider the atomistic theory and relevant experiences, starting just before the nineteenth century, introducing the modernity of quantum mechanics and relativity. Among them, the powerful observation of the drift of electrons in vacuum when submitted to an external electric potential [13] has a strong similarity with the flow of a river under gravity. The electric case has the advantage of a possible control of the flow direction by means of the choice of the external electric field direction. This situation can be also compared successfully with the effect of a common opinion in sociology or in politics with the massive effects which follows this mere control. Of course, this common opinion can be issued from different generative sources, some leaders, some discoveries for instance. Quite similarly to the case of the electronic drift, the social consequences can be strong and astonishing.

The practical applications of this easily forced effect have been already quite numerous according to progressive technical advances from electronic and ionic transport in vacuum, in gas or in solid semiconductors, with the basic examples of electronic tubes, electronic semiconductor components, television, and further to come according to miniaturization and cost reduction. 
The main trick in these quite numerous applications lies in the common use of a control bait, a grid, in order to define the flux direction without nearly any energy consumption, a nearly free control of a large flux involving a massive energy. This simple trick is the basis for non-linear detection at a threshold and for amplification or oscillation, in the language of electronics and informatics.

\subsubsection{Mixed concepts in hard sciences and human sciences}

In politics, or in general communication, this concept of an easy drive of a large mass means to strike a sensitive chord, even with the risk of inducing a potential crisis. In electronics feedbacks allow to stabilize such regimes. In politics couplings are quite more complex with less control and crisis often occurs! This basic non-linear effect is, at least, highly suggestive for quite different disciplines, each one with its own properties and dynamics.

\section{Complexity and disorder as an interdisciplinary example}

As already noticed complexity and disorder are common unavoidable features in all disciplines. So, the present problem is more concretely to show how complexity and disorder are treated in each discipline and the question is: "Are they common approaches to this problem?" A complete answer cannot be done. But simple lines of convergence can be drawn. A first point consists in looking at complexity over several scales for a given discipline and comparing these results together. This is already a whole program! A further question concerns the limiting scale: "Are there also complexity and disorder at the very individual level?

\subsection{Complexity and disorder at several scales}

The quite general idea of observing facts at different scales, with the underlying question "Are observations similar or not at different scales?" has been quite fruitful in several disciplines, with the Nobel prize for the proposal of the Renormalization group [14] for instance, and with now famous fractals [15]. Here we just want to give a concrete flavour of such multi scales observations by considering a few examples.

The already introduced theme of leadership by a very reduced community acting without energy balance, basically a social effect, has, backward, many applications in different disciplines. The restriction of this domination over a more or less small group, at different scales, enables to make a clear analysis.

In physics, from the case of impurities acting as colour centres for optical applications up to the case of magnetic impurities with Kondo effects on magnetoresistance, the effect of size reduction up to nanoparticles is quite promising when avoiding the necessity of averaging. The use of smaller and smaller nanoparticles enables to avoid the unfortunate randomness effect, introduced by the requirement of considering a meaningful signal for large samples with multiple defects. In the very case of simple miniaturized samples, the effect of one single impurity can be observed. This successful process restricting the noisy effect of randomness is already used. Single colour centres were recently observed within a nanoparticle [16] but the case of single Kondo defects remains to be observed. In large samples Kondo effects are known to lead to spin glass effects, a complex situation [17], since these magnetic defects, impurities are randomly arranged within the macroscopic sample. As a matter of fact, intermediate sizes are also interesting. So, the observation of a single magnetic impurity in a nanoparticle, a single Kondo effect would avoid a part of this disorder.

Reversely, the study of leadership within a restricted sample, a restricted community sounds to be interesting, not only in politics, but also in microsociology as well as in ethnology. For instance, Indian tribes in central and south America are quite restricted. Their social organization has been well observed with many such individual examples $[18,19]$. They also share some meetings between a few tribes from time to time. One interesting concept revealed by these studies on small tribes is the incest prohibition and its mathematical consequences in the social organization [20], a rather unexpected result of the study of these prime civilizations which reveals a high degree of potential mathematical formalism! This basic structuring incest prohibition law was also studied in larger scale units, even if direct its appearance is less obvious.

The comparison between local and global effects is thus useful between different parts of several disciplines such as physics and anthropology. The parallel field of microsociology [21] also revealed very local phenomena of social organization which are less obvious when looking at a larger scale.

\subsection{Complexity and disorder at the individual level}

In many disciplines, complexity means multiplicity. This is the case in quantum mechanics where the wavy character of the wavefunction means non-localization and so multiple positions at any time. This paradoxical situation led to a considerable amount of work [22], with a full richness of interpretations.

In usual life as well as in history, the usual word "main stream" evidences this multiplicity of other streams which cannot be strictly neglected and strongly contribute to events with their complexity. This internal complexity is essential to human sciences [23] since it brings variability, potential variations.

Even at the individual level of a person, the multiplicity of parts is obvious. The genetic characters explicit a correspondence with quite numerous ancestors and so quite numerous possible features. The human activity means quite numerous meetings, quite numerous friends, quite numerous colleagues, quite numerous influences. Even if Pascal restricted the number of possible friends to four [24], modern sociology shows that the average number of influent persons for a single one is of the order of several tens at a given time [25]. When looking on a 
larger time scale, several hundreds of connected people must be considered. This multiplicity of potential influences at the individual level is responsible for the very individual behavioural complexity, unpredictability! So, there is no ultimate level for complexity.

\section{Conclusion}

In this short introduction, the interest for pluridisciplinary meetings with the common theme of complexity and disorder was shown. In the main part of this introduction, common approaches based upon observations at different scales were underlined. This common concept about the open question of scale invariance already introduces a basic linguistic feature and a few questions. Are they similar concepts in different disciplines? How these more or less similar concepts are expressed in different disciplines? And which are the corresponding words for these concepts in each discipline? So, the real challenge of these meetings is to introduce a common thinking in quite different disciplines.

The practical classification of these articles given in the Complexity disorder meetings consists here in starting from philosophy and human sciences, before mathematics, physics, chemistry and medicine

As organizer of these meetings and of this review, I am glad to acknowledge the constant help of the Physics department of University Paris Diderot which is now a part of "Université de Paris". It is also my pleasure to acknowledge the practical help of three laboratories of this University which are linked to this department called "UFR de Physique". These laboratories are respectively, LIED "Laboratoire Interdisciplinaire des Energies de Demain », MPQ « Matériaux et Phénomènes Quantiques », and MSC « Matière et Systèmes Complexes » .

\section{References}

1. E. Durkheim, De la division du travail social (PUF, Paris, 2013)

2. P. Ghewy, M. Jaillot, A. Tchekemian, in L'avenir de la complexité et du désordre, 341 (Editions Matériologiques, Paris, 2018)

3. A. Comte-Sponville Le bonheur, désespérément (Librio, Paris, 2018)

4. G.W.F. Hegel, Aesthetics (Clarendon Press, Oxford, 1975)

5. K. Popper, C.J. Eccles, The self and its brain (Springer, Berlin, 1977)

6. P. Duwez, J. Appl. Phys. 311136 (1960)

7. A. Tanguy, in L'avenir de la complexité et du désordre, 171 (Editions Matériologiques, Paris, 2018)

8. Plato, Timee, (Les Belles Lettres, Paris, 1949)

9. R. Callinger, A contextual History of Mathematics: To Euler (Upper Saddle River, Prentice-Hall, 1999)
10. F. Bacon, Novum organum (Hachette, Paris, 1857)

11. I. Newton, Philosophiae Naturalis Principia Mathematica (London, 1687)

12. S. Berthier, in Complexité et Désordre (EDP Science, Les Ulis, 2015) 63

13. N.W. Ashcroft, N.D. Mermin, Solid state physics (Saunders College, Fort Worth, 1955)

14. K.G. Wilson, Phys. Rev. B 43174 (1971)

15. B.B. Mandelbrot, The fractal geometry of nature (Macmillan, New York, 1983)

16. J.-P. Tetienne, T. Hingant, L. Rondin, S. Rohart, A. Thiaville, J.-F. Roch, V. Jacques, Phys. Rev. B 88 214408 (2013)

17. S.F. Edwards, P.W. Anderson, J. Phys. F 5965 (1975)

18. C. Levi-Strauss, Tristes Tropiques, (Plon, Paris, 1955)

19. P. Clastres, Chronique des indiens Guayaki, (Plon, Paris 1972)

20. C. Levi-Strauss, La pensée sauvage, (Plon, Paris, 1962)

21. H.S. Becker, Art worlds, (University of California Press, L.A. 1982)

22. H. Zwirn in this volume (EDP, Les Ulis, 2020)

23. N. Piqué in L'avenir de la complexité et du désordre, 69 (Editions Matériologiques, Paris, 2018)

24. B. Pascal, Pensées, (Garnier Flammarion, Paris, 1670)

25. C. Bidart, A. Degenne, M. Grossetti in this volume (EDP, Les Ulis, 2020) 